\title{
Axion N and Photoluminescence
}

\section{Ogluzdin VE*}

Federal State Budgetary Institution A.M. Prokhorov, Moscow, Russia

*Corresponding Author: Ogluzdin VE, Federal State Budgetary Institution A.M.

Prokhorov, Moscow, Russia.
Received: August 25, 2021

Published: September 15, 2021

(C) All rights are reserved by Ogluzdin VE.

\section{Abstract}

A photoluminescence model is proposed that takes into account the role of axions, which are associated with the width of the photoluminescence spectra; the lifetime of axions has been estimated for a number of media.

Keywords: Photoluminescence; Axion; Dispersion Medium

\section{Introduction}

The paper considers the role of axion [1] in the process of photoluminescence (PL) in dispersive media (DS) in the spectral region $0.5-3 \mathrm{eV}$. Purpose of the work: based on the analysis of experimental data, to propose a model reflecting the contribution of axions to the PL process.

Axion

From the hypothesis of Henry Primakov (Primako ff) [2] it follows that because of the decay of an axion in the field of the atomic nucleus of an atom placed in, the creation of two new photons in an elementary act is a necessary and sufficient condition for the existence of an axion. The Primakov effect can be direct or inverse. The straight line corresponds to the fusion of two photons in the field of an atomic nucleus, leading to the creation of an axion.

In this work, we are not limited to analysing our own results $[3,4]$. Comparison of number of publishing $x$ are experimental $x$ DATA $x$ obtained groups of researchers in the visible and nearinfrared regions with pektra, allows tons of new insights into the process of the PL observed in DS.
For reference, we point out that in theoretical physics there is no consensus on the nature of the axion. At this stage, all that matters to us is that the mass of the axion is small. This fact corresponds to the statement: "in the modified theory within the framework of the Grand Unification... the axion must be... a particle of small mass" [1], comparable in size to the small mass of a moving photon. Therefore, it becomes possible to extract information about the presence of axions in certain optical processes initiated by photons. We will be guided by this circumstance in the subsequent part of the work.

On Pisani the optical phenomena using wave representations do not allow to fully implement the interpretation of the results observed in the junction of the optics and elementary particle physics. Involving the methods used in the physics of the quantum world can help solve the problem. If we turn to photons (quanta), which have successfully moved from the physics of elementary particles to optics with the advent of lasers, then the connection between optics and the physics of the quantum world turns out to be real. This fact can help to clarify the nature of the axion. In spumoni to generate optical radiation of harmonics, optical parametric generation. The authors of [5], the subtitle of which is "electromagnetic 
waves in nonlinear... media", argued that when using the quantum interpretation, the creation of harmonics and the appearance of combination frequencies indicate an essential role of the processes of fusion or fission of photons (light quanta), while noting the need for taking into account the laws of conservation of energy and momentum in nonlinear optical transformations. When the second harmonic is generated in an elementary act, two photons of the pump radiation merge. In a parametric light generator [6], the decay of a pump photon in an elementary act ensures the appearance of two photons at the output of a nonlinear medium: signal and idle.

The analysis of the results obtained in studying the PL of silicon nanoparticles suspended in ethanol [7,8], in the author's opinion, allows the presence of axions. As a rule, the medium under study is a DS (glass, crystal, or liquid), in which the pump radiation is converted, leading to the appearance of PL quanta. The process of conversion of pump radiation is associated with the production of axions and their decay. The energy of PL quanta, as a rule, differs from the energy of the pump radiation quanta.

From chemistry [9], we should recall the DS, in which a certain phase in the form of small formations is distributed in the main volume of the continuous phase. In our work we will deal with a DS, the structure of which can be represented as follows: continuous phase - glass, crystal structure, liquid. The second component of the DS: atoms or nanoparticles of the alloying material distributed (suspended) in the volume of the continuous phase: bismuth, silicon. And finally, the third phase: the electrons of the atoms (or nanoparticles) of the alloying material.

Let us also recall the complex environment (CS). CS is a substance, which includes complex particles (atoms or nanoparticles) capable of independent or coherent existence in a liquid, amorphous (glass) or crystalline environment, which plays the role of a matrix. The complex particle itself, in turn, can be formed from other, simpler particles (nuclei surrounded by electrons). In optics, it is natural to talk about DS - a medium that is associated with the phenomenon of light dispersion. These are, for example, glasses doped with bismuth atoms.

It is known that when monochromatic radiation is used for pumping a DS, the PL spectrum is, as a rule, broadened. The PL spectrum width is undoubtedly associated with transitions in the atom of the dopant. Among the reasons for the broadening of the PL spectrum are: the number, finite width of electronic levels participating in the elementary act of excitation of atoms of the element used for doping the medium, shifts of these levels in the pumping field [10], temperature conditions of the experiment.

To clarify the definition of FL, it is necessary to introduce an idea of the virtual level. The virtual level, the energy of which is determined by the sum of the energies of two pump quanta, as a rule, does not coincide with the levels of the atom of the substance used for doping the medium. His life time is extremely limited. According to Primakov [1], the addition in an elementary act of two quanta of pumping radiation in the field of the atomic nucleus of the dopant atom can lead to the creation of an axion. Virtual level instability due to the interaction of the light beam of photons with a plurality of layer s atom alloying element leads to disintegration axion s to couple quantums.

The energy of one of them is spent on heating the medium due to nonradiative relaxation. Unfortunately, as a rule, the issue of heating and transfer of heat to DS is little discussed. But when dye lasers were being developed, the issue of heat removal was the main one.

The second quantum of the decayed axion propagates through the DS with a slower velocity, which depends on the structure of the energy spectrum of the atoms of the alloying element, its energy, i.e. frequency. In [11], when studying the Vavilov - Cherenkov effect, it was shown that the speed of a photon in such media is phase. Let us recall the multitude of harmonic oscillators (electrons) of different frequencies encountered on the path of a photon, and the dispersion law for each of them [12-14].

At the output of the DS, we obtain, as a rule, a broadened PL spectrum. For example, upon excitation of silicon nanoparticles embedded in ethanol by argon laser radiation [7,8], a PL spectrum with a width from $464 \mathrm{~nm}$ to $670 \mathrm{~nm}$ was obtained.

What can the width of the PL spectrum give us, and what information can we extract from this?

Due to the quantum-mechanical uncertainty of the position of the energy levels of the atom, we have the right to believe that the width of the PL spectrum recorded by the photodetector corre- 
sponds to the natural width [15]. If $\tau$ is the average lifetime of the axion at the virtual level, $\Delta \mathrm{W}$ is the energy of this level, then for this situation the known uncertainty relation holds

$\Delta \mathrm{W} \tau \approx \mathrm{h} / 2 \pi \cdot---(1)$

According to [16], the uncertainty relation, due to the smallness of $h$, is essential only for microsystems, which we are dealing with. We will use this relation to determine the lifetime of the axion.

The broad PL contour I ( $v$ ), which reflects the dependence of the PL power on frequency, usually has a maximum (hump) and falling wings. For the width of the spectral line $\Delta \mathrm{W}$, the frequency difference is taken, which corresponds to a halving of its peak power I $(v)$ ${ }_{\max } / 2$. We Consider it a few examples to illustrate the relationship of the spectral width of the PL emission band and the time of the axion lifetime.

To estimate the lifetime of the axion, the following relationship was used: $1 \mathrm{~cm}^{-1}=2.99793 \cdot 10^{10} \mathrm{~s}^{-1}(\mathrm{~Hz})$. The lifetime of the axion, which determines the width of the PL spectrum, is much shorter than the lifetime of the excited energy levels of the doping atoms, which, according to reference data [17], corresponds to a value of the order of $\tau_{0} \sim 10^{-8}$ sec.

The calculation shows that for the case of silicon nanoparticles suspended in ethanol $[7,8]$, the axion lifetime is estimated as $\tau \sim$ $3.48 \times 10^{-13}$ sec.

If alloying Bani glass ASIC 1 zovalsya bismuth while axion life is estimated to be: $\mathrm{T} \sim 3.48 \times 10^{-13} \mathrm{sec}$. [18], $\tau \sim 2.93 \times 10^{-13} \mathrm{sec}$. [19], $\tau \sim 3.64 \times 10^{-13}[20]$.

\section{Photoluminescence}

To clarify the concept of FL, let us first dwell on the definition of this process. According to the Encyclopaedic Dictionary of Physics in 1984 [17] PL - this luminescence, excited by optical radiation. Remaining within the framework of this definition, it is necessary to consider three options: 1) resonant radiation, 2) PL corresponding to the Stokes rule, 3) anti-Stokes PL. All options obey the law of conservation of energy. According to [21], the law of conservation of energy for PL has the form:

$\mathrm{h} v_{\mathrm{pf}}=2 \mathrm{~h} v-\mathrm{h} v_{\mathrm{ij}},-----(2)$ g de hv - energy quanta (photons) of the pump - the light radiation used to excite photoluminescence; quantity $v$ is the frequency of this radiation.

$\mathrm{h} \nu_{\mathrm{ij}}$ is the energy spent on nonradiative relaxation.

The indices ij correspond to the set of transitions involved in the relaxation process; According to (2), each of these transitions corresponds to a component in the PL spectrum at the output of the medium. The quantity $v_{\mathrm{ij}}$ is a set of many transition frequencies associated with nonradiative relaxation and transfer of thermal energy to the medium. Since the PL spectrum in a DS is usually broadened, it is natural to assume that the PL process is accompanied by a multitude of electronic interlevel transitions in the atoms of the element used for doping the DS, which are responsible for the nonradiative relaxation. Naturally, for each frequency component $v_{\mathrm{pf}}$ of the broadened PL spectrum, the energy conservation law is satisfied: $h v_{\mathrm{pf}}$ is the PL photon energy, and $v_{\mathrm{pf}}$ are the frequencies filling the broadened PL spectrum. The "pf" index (from "photoluminescence") corresponds to the entire set of frequencies involved in PL. The index "ij" corresponds to the same number of frequencies of nonradiative transitions.

It follows from relation (2) that the value of the pump radiation frequency is the arithmetic mean between each pair of frequencies $v_{\mathrm{pf}}$ and $v_{\mathrm{ij}}$

Pezonansnoe radiation - the simplest case of the PL. In this case, we have the implementation of Einstein's scheme [14]: 1) absorption of a light quantum, 2) spontaneous emission of a quantum, and 3) stimulated emission of a quantum at a frequency $v_{\text {pf. }}$ We should recall the experiments of the American optician R. Wood, who studied the resonant interaction of light radiation in a cell with atomic sodium vapor in the frequency range of the main doublet. The process of absorption by sodium atoms of quanta incident on the window of a cell with atomic vapours is accompanied by processes of spontaneous and stimulated resonance radiation.

Note that, in contrast to the resonance case, the PL spectrum at the exit from the DS is, as a rule, broadened. Most often, it is broadened with respect to the pump frequency into the Stokes region of the spectrum. This fact is considered to be fundamental for determining PL. We will return to this issue below. This was noted 
by Paul R.V. [14], referring to the experiments of SI Vavilov [22]. And, finally, the law of conservation of energy is unambiguously related to the rule of mirror symmetry known in PL between the PL spectrum and the absorption spectrum [23]. Indeed, from the energy conservation law (6) for the frequencies involved in the PL process, for the Stokes region of the PL spectrum, we have the mirror symmetry

$v_{\mathrm{ij}}-v=v-v_{\mathrm{pf}},----(3)$

where $v>v_{\text {pf }}$

A similar relationship can be written for the anti-Stokes region of the PL spectrum:

$v_{\mathrm{pf}}-v=v-v_{\mathrm{ij}},----(4)$

Where $\mathrm{N}<v_{\mathrm{pf}}$

Relationships (3.4) helped to understand the PL spectra presented in [7] or borrowed from materials of various authors, in which the PL results are presented. Unfortunately, the accuracy of frequency estimation at the stage of analysis of PL spectrograms in DSs published by various researchers is not high enough. However, it is possible to establish coincidences between the tabular values of the wavelengths or frequencies responsible for nonradiative relaxation and the values of the frequencies or wavelengths calculated from the results of the experiment.

Let's go further. The energy conservation law (2) yields a linear relationship between the pump radiation power and the PL radiation power at the DS output. This is confirmed by the results of a number of studies presented in works on PL in optical fibers [1820].

\section{Conclusion}

We have already said that the definition of PL in DS needs to be supplemented and clarified. We preliminarily stopped at the definition according to which PL is a glow generated by optical radiation. According to S.I. Vavilov luminescence is an excess of radiation over temperature, provided that the excess radiation has a finite duration that exceeds the period of light oscillations. Stokes' law states that PL light always has a longer wavelength compared to the radiation wavelength used to excite PL. According to Lommel, the PL spectrum as a whole and its maximum are always shifted in comparison with the excitation spectrum and its maximum towards long waves.

For a long time, it was believed that a substance is characterized by a certain PL spectrum, which is insensitive to changes in the wavelength of the exciting light. As we can see, this rule is violated. Experiments in silicon -, bismuth-doped media Confirm zhdayut this position.

The validity of the established by V.L. Levshin rules for mirror symmetry of absorption and PL spectra, which follows from the definition of PL considered in this work.

\section{Bibliography}

1. F A Wilchek, UFN. 175, 1305. [the F. A. Wilczek, Proceedings of the National Academy of Sciences of the United States of America 102 (2005): 8403.

2. S Sikivie., et al. Physical Review Letters 98 (2007): 172002.

3. In. E. Ogluzdin. Engineering Physics 9 (2015): 16 (2015)

4. VE Ogluzdin. Scifed Publisher Journal of Nuclear Science 3.1 (2019): 1 .

5. CA Akhmanov P. In. Khokhlov, Problems of Nonlinear Optics. 1962-1963 (M. AS C SSR, Institute of Scientific Information (1964).

6. CA Akhmanov P. In. Khokhlov, Quantum Electronics. 1, 1022 (1974) S. A. Ahmanov, R. The V. Hohlov, Soviet Journal of Quantum Electronics 1 (1975).

7. VE Ogluzdin. Brief communications on physics. M.; LPI, 9, 3 (2002) V. E. Ogluzdin, Bulletin of the Lebedev Physics Institute. N.- Y.; Allerton Press. 9 (2002): 3 (2002).

8. VE Ogluzdin. Izvestiya RAN. Physical Series 70 (2006): 418.

9. HH Glinka. General Chemistry. M., High School (2003).

10. VE Ogluzdin. Laser spectroscopija. 16.8 (2006): 1178.

11. The. E. Ogluzdin, ZhETF. 79, 361 (1985) [V. E. Ogluzdin, Sov. Phys. Zhurnal Eksperimentalnoi i Teoreticheskoi Phiziki. 52.2 (1980): 181.

12. In. Ditchburn, Physical Optics. M., Science. R. The W. Ditchburn, Light. London, Glasgow, Blackie and son LIMITED (1963). 
13. FA Korolev. Theoretical Optics. M., Higher school (1965).

14. B Paul. An Introduction to Optics. M., A., Ogiz, Gostekhizdat [ R. Bed and. POL, Introduction to optic (1947).

15. H. Seidel., et al. Technique and Practice of Spectroscopy. M., Science (1972).

16. With. Davydov, Quantum Mechanics. M., State Publishing House of Physics and Mathematics. literature (1965).

17. Physical encyclopedic dictionary M., Council. Encyclopedia (1984).

18. The V About. Sokolov., et al. Journal of Physics D: Applied - IOPscience 42 (2009): 095410.

19. AA Krylov., et al. Quantum Electronics 39.1 (2009): 21.

20. By. E. Ryumkin., et al. Quantum Electronics 44 (2014): 700.

21. In H Levshin. Photoluminescence of Liquid and Solid Substances. ML Gostekhizdat (1951).

22. With And. Vavilov, Microstructure of Light. M.; Publishing house of the Academy of Sciences of the USSR (1950).

Volume 5 Issue 10 October 2021 (C) All rights are reserved by Ogluzdin VE. 